\title{
Building Consensus via a Semantic Web Collaborative Space
}

\author{
George Anadiotis \\ IMC Technologies \\ Athens, Greece \\ ganadiotis@imc.com.gr
}

\author{
Konstantinos Kafentzis \\ IMC Technologies \\ Athens, Greece \\ kkafentzis@imc.com.gr
}

\author{
John Pavlopoulos \\ IMC Technologies \\ Athens, Greece \\ ipavlopoulos@imc.com.gr \\ Athens University of \\ Economics and Business \\ Athens, Greece \\ annis@aueb.gr
}

\author{
Adam Westerski \\ Universiadad Politecnica de \\ Madrid \\ Madrid, Spain \\ westerski@dit.upm.es
}

\begin{abstract}
In this paper we outline the design and implementation of the eDialogos Consensus process and platform to support wide-scale collaborative decision making. We present the design space and choices made and perform a conceptual alignement of the domains this space entails, based on the use of the eDialogos Consensus ontology as a crystallization point for platform design and implementation as well as interoperability with existing solutions. We also present a metric for calculating agreement on the issues under debate in the platform, incorporating argumentation structure and user feedback.
\end{abstract}

\section{Categories and Subject Descriptors}

H.4.2 [Information Systems]: Types of SystemsDecision support; H.5.3 [Information Systems]: Information Interfaces and PresentationGroup and Organization Interfaces; J.4 [Computer Applications]: Social and Behavioral Sciences

\section{General Terms \\ Computer Supported Collaborative Argumentation}

\section{Keywords}

Collaborative Decision Making, Issue Based Information Systems, Argument Mapping, Argumentation, Citizen Engagement, Consensus, Alignment, Process, Platform, Metrics

Permission to make digital or hard copies of all or part of this work for personal or classroom use is granted without fee provided that copies are not made or distributed for profit or commercial advantage and that copies bear this notice and the full citation on the first page. To copy otherwise, to republish, to post on servers or to redistribute to lists, requires prior specific permission and/or a fee.

SWCS 2012 Lyon, France

Copyright 20XX ACM X-XXXXX-XX-X/XX/XX ...\$10.00.

\section{INTRODUCTION}

In recent work, we have examined Virtual Communities as emergent socio-technological phenomena and shown how the increasing level of connectedness among people via the use of ICT - what is termed Social Media (SM)- has both catalyzed and been catalyzed by this tendency. Furthermore, we have examined how SM empower Virtual Communities, how Semantics empower SM as knowledge elicitation platforms leading towards the Enterprise2.0 and Government2.0 paradigms and how we can leverage these phenomena via Open Innovation to build a structured deliberation process that goes beyond open, towards inclusive policy making [3].

In this paper, we present the eDialogos Consensus Building platform, an initial implementation of the outlined Open Innovation structured deliberation process: social semantic software with the mission to bring together human agents and software agents in order to foster knowledge-intensive collaboration via content creation and management. In other words, a Semantic Web Collaborative Space with the ultimate goal of promoting collective decision making.

The rest of the paper is structured as follows: we begin by presenting the background and rationale for the design of the eDialogos Consensus Building platform in Section 2, and present the platform and its functionality in Section 3. In the following sections, we focus on the unique aspects of the platform, namely the Open Innovation Semantic Web infrastructure for collaborative decision making (Section 4) and the Consensus Building Model (Section 5). Finally, we conclude and present future work in Section 6.

\section{BACKGROUND AND RELATED WORK}

In order to trace the roots of ICT-supported collaborative problem solving, we have to revisit the IBIS methodology [11]. As IBIS was one of the first approaches designed explicitly with the goal of being applied using information systems, inevitably all subsequent collaborative problem solving approaches somehow reference it. IBIS' success lies in the fact that it provides a structure and a process simple to conceive and use, and yet powerful enough to express 
argumentation cycles in groups. Initially, the general area in which issues exist is identified. Within this area issues are identified through discussion, and each is recorded as a question. Possible answers to the question are identified as positions and then participants express arguments in favour of or against a position. Unravelling issues in this fashion may lead to an answer on which all participants agree, in which case the process terminates, or if this is not the case then the positions and arguments are re-examined -wherever possible treated as issues themselves- and the decomposition cycle repeated.

The system was originally described in terms of a model and algorithms, although at the time of writing the procedures were implemented largely by hand. This was of course a major shortcoming of IBIS, but in the following years a number of tools to support the process have been developed. This development has taken on a couple of defining characteristics, namely formal argumentation and argument mapping, that lead to the creation of a new tool genre. This tool genre is an offsrping of gIBIS, a sophisticated, hypertextbased graphical tool for using the IBIS techniques, which later led to the development of the commercial QuestMap tool, in turn superceded by Compendium [12]. Other tools in this genre are the recently introduced PolicyCommons [7], based on the Cohere tool [26], and the Carneades tool in its latest incarnation [14].

The aforementioned tool genre is used mainly in the domain known as ICT for Governance and Policy Modelling (IGPM). IGPM is traditionally thought of as being comprised of two complementary yet separated research fields: the Governance and Participation Toolbox, including technologies for mass conversation and collaboration (Social Networking, Web2.0/3.0 and Crowdsourcing approaches) and the Policy Modelling domain including technologies such as Process Modelling, Visualization, Gaming, Mixed Reality and Simulation [1]. This divide is evident in the above mentioned tools and their characteristics. Since formal argumentation is approached based on complex logic contructs, suitable for legal argumentation but hardly approachable for average users [29], while argument mapping is based on GUI elements for interactions, most tools tend to:

- Focus on the argument mapping aspect, making for tools that are user-friendly but offer limited functionality - glorified mind maps.

- Focus on the argumentation grounding aspect, making for a complex and unappealing user experience - made by and for argumentation experts.

So in terms of striking a balance between expressivity and real-world applicability, it seems that the Cope_It tool [16] with its (limited) support for import from existing forums, the Deliberatorium tool [17] aiming at large scale argumentation and the widely acclaimed DebateGraph ${ }^{1}$ tool are as close as it gets at this point. For an in-depth review and analysis of the domain, the interested reader may refer to [25].

An obvious shortcoming that spans these tools is lack of interoperability: although the overlap in shared process and concept space is striking [13], it's not until recently that the interoperability effort made its first steps [22], covering only

\footnotetext{
$\overline{{ }^{1} \text { http://www.debategraph.org }}$
}

the syntactic aspect while ignoring the semantic one. A proposal in this direction has been put forth [5], but it seems to remain dormant and is not used in any of the tools. This is rather surprising, if we consider that "IBIS in effect covers knowledge elicitation, modelling and reasoning. The use of atomic statements and relationships between these statements anticipates some of the core techniques of Semantic Web developers three decades later" [5].

Another related effort for semantic grounding and interoperability is expressing the AIF argument exchange format as an ontology [23], in support of a vision to lay the foundations for a World Wide Argument Web that remains unfulfilled up to this day. In addition, by following the AIF format the resulting ontology is also built mainly to support the expression of argumentation schemes, resulting in a rather complex schema. There is also the SWAN ontology, which includes elements of scientific discourse and argumentation. Even though much simpler than its AIF-based counterpart though, this ontology still takes a formal approach to argumentation. This results in a schema appropriate to support scientific research with hypotheses and supporting evidence (this was its intended use in fact), but does not lend itself very well to representing argumentation on wicked problems by a large number of stakeholders [10]. Finally, OPOL expresses the representation of political programs as an ontology [6], which while being useful in its own right does not say anything about the argumentation and process of decision making.

A crucial aspect missing from the aforementioned tools is support for incorporating user feedback in the decision making process. The assumption seems to be that laying down the arguments and/or providing a structured process will facilitate the decision making in one of two ways:

- By resulting in an enlightened understanding of the problem on behalf of the participants, who will somehow reach a decision via offline procedures - typically relying on the role of the facilitator, an individual skilled in the process, the tools and able to communicate effectively with all participants.

- By documenting all the arguments, their logical premises and structure, so that applying reasoning rules will enable tools to provide the 'algorithmically optimal' solution.

Unfortunately, neither assumption holds when applied to wicked problems with a big and diverse group of participants and stakeholders, as most real-life public sphere issues tend to be.

While evidence shows that documenting arguments in a structured way is a very effective knowledge elicitation mechanism [17], and domain knowledge certainly promotes decision making capabilities, everyone cannot be an expert in every domain. But even if they could, this alone would not make for an ideal decision making situation (cf [15]), or provide the means to capture the input required for decision making. Relying solely on formal argumentation and modelling on the other hand does not resonate well with stakeholders, as the underlying modelling typically used does not have a history of sucess that would make it trustworthy and is also quite different from the behavior observed in human societies [1]. Therefore, it promotes the already existing feeling of disconnectedness from the public sphere. 
An approach that tries to address this is Citizen Engagement (CE). CE builds on both ICT and political science and bears the promise of reconnecting citizens and all other stakeholders to decision-making and governance, central or local, by means of policy formulation and evaluation. The ultimate goal however is to strengthen and empower democratic governance at all levels of society and to promote individual citizen evolution as part of the collective process in a deliberative, discursive way. Ironically though, all technical and legislative issues aside, what remains the greatest challenge in today's CE landscape is actually achieving substantial CE levels [21].

Since at its foundation, SM is a set of technologies and channels aimed at forming and enabling a potentially massive community of participants to productively collaborate, it seems that CE and SM have common ground and a way forward for CE could be to learn from and adopt the SM paradigm. Experience with such systems has shown that they can foster, by virtue of reducing the cost of participation, voluntary contributions at a vast scale, which in turn can lead to remarkably powerful emergent phenomena, such as idea synergy, the long tail, many eyes and wisdom of the crowds [17].

These emergent phenomena manifest themeselves in emergent communities. Traditionally, CE efforts are associated with governmental organizations, as they are the ones with the means and the legitimacy to sponsor such projects. Lately the increasing need for self-governance in emergent communities, potentially geographically dispersed and typically communicating over SM, has given rise to a new genre of decision-making approaches. These approaches range from the simple 'outsourcing' of functions of traditional politics, such as campaigning [8], to a SM platform, through the very use of SM capabilities in ways that redefine the decision making process itself, such as expertise and trust-based vote delegation [27, 24], grass-roots decision making infrastructure [2], or open innovation deliberation [3].

However, while SM practices may indeed provide benefits, since existing SM are not designed for CE they suffer from a number of drawbacks that render them inappropriate for this purpose, namely disorganized content, low signalto-noise ratio, quantity rather than depth, polarization and dysfunctional argumentation [17]. What is needed therefore is a new genre of tools that will stand in the middle ground between completely unstructured, general purpose SM and highly structured, formal argumentation tools. The goal would be to harness the power of user-generated content, feedback and interaction via a process and platform that provides only as much structure as necessary, without being a burden to learn and use.

In this respect, the use of metrics to estimate exceptions has been proposed: exceptions in this context are situations that potentially require user intervention or are interesting for users [17]. The approach tries to identify these exceptional situations qualitatively and then model them as a metric that expresses the expression quantitativaly, based on available data. The rationale for this kind of metrics, introduced in [17], is to direct user attention to topics and events best-suited for them, picked from a potentially vast collaboration space. However, no concrete metrics are presented. In [19], a metric for evaluation of argument strenght is introduced, however it is purely theoretical (based on game-theoretical modeling) and based on the assumption that different arguments on a topic could somehow be assigned a strenght factor through some unidentified process. Finally, in recent work [18] the notion of Social Abstract Argumentation is introduced, defining a framework to take user feedback via SM into account when calculating argument strength.

\section{A DELIBERATIVE, DISCURSIVE MODE OF DECISION MAKING: THE EDIALO- GOS CONSENSUS PROCESS AND PLAT- FORM}

Based on the domain analysis of Section 2, we have embarked to design a process and a platform for a deliberative, discursive mode of decision making that incorporates bestof-breed characteristics and tools from each discipline and approach. Our work has been inspired by existing methodological and technical background, while at the same time incorporating experience gained through application of deliberative $\mathrm{CE}$ processes in a number of real-world settings such as local ${ }^{2}$ and regional ${ }^{3}$ government or mainstream political parties ${ }^{4}$. We documented a theoretical framework and established a structured deliberation process [3], utilizing Semantic Web Technology to facilitate dialogue [4] and the Open Innovation principles [9].

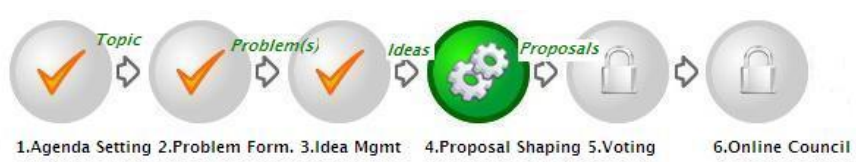

Figure 1: The Open Innovation deliberation process

Our priorities in this process were:

- To make the entry barrier for users as low as possible. This lead us to design the system as a SM platform to make for an intuitive user experience, incorporating well-known tools such as one-click content creation and reaction facilities, groups for sharing and collaborating with others on specific topics, activity streams for keeping up to date with user actions and reactions and different ways of organizing, navigating and locating content and users, such as hierarchical structure, horizontal association and universal search.

- To build on and maintain compatibility with existing approaches. This lead us to adopt the IBIS methodology, as the one that underpins a wide array of existing approaches and is well known, well understood and uses a simple yet powerful notation that enables the expression of collective decision making as a graph that can be documented and explored. Furthermore, in order to align approaches and systems, we provide a way to conceptually document alignment as well as facilitate interoperability on the technical level, by means of an ontology.

\footnotetext{
${ }^{2}$ http://epractice.eu/en/cases/edialogosawards

${ }^{3}$ http://www.samos-dialogos.gr

${ }^{4}$ http://www.nd.gr/web/dialogos
} 
- To enable, encourage and make use of usergenerated content and feedback in every phase of the process. Using tools that facilitate the mechanics of user engagement and pairing them with a design that makes for an intuitive user experience is a prerequisite to achieve this goal. Going one level beyond this however means designing and implementing a model that estimates argument strength and agreement level based on user feedback. Furthermore, a model of participation as a strategic game that rewards contributions and engagement would further promote the process.

The opportunity to apply this process in practice and deploy the platform presented itself when we were contacted by the European Academy of Allergy and Clinical Immunology $\left(\mathrm{EAACI}^{5}\right)$, with the aim of implementing a process through which its 7000 members would be enabled to collaborate in a distributed fashion in order to collectively author medical guidelines. In order to sucessfully apply the process in the context of decision making taking place in EAACI medical guidelines formation, we had to consult with EAACI members and adjust the process as needed. The Open Innovation eDeliberation process is a tight 'serial process' within a specific time-frame, with 6 discrete phases embedded in each deliberative cycle, depicted in Fig 1.

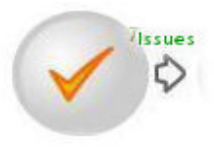

Problem Formulation

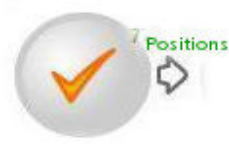

Idea Management

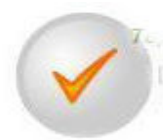

Voting
Figure 2: The eDialogos Consensus deliberation process

Initially we have the agenda setting phase, in which participants may choose among a list of proposed topics for deliberation, or propose their own topic. After having chosen a general topic for the deliberation in the agenda setting phase, we break down this topic in specific problems to address. Problems must be clearly formulated in order to be addressed, and this process is supported by appropriate tools. The outcome of the problem formulation phase will be a number of problems, therefore an idea management phase follows in the process in order to collect ideas for dealing with each problem. After the idea management phase concludes, a proposal shaping phase is initiated, with the aim of composing and refining ideas into well-rounded proposals for adoption by the hosting organization. Finally, the voting phase on completed proposals gives participant input for the decision making that takes place in the Online Council phase, determining proposal adoption, implementation and deployment.

In the case of EAACI, the agenda setting phase was omitted. This phase is essential when the decision-making domain is very broad, as is the case for political decision making, but for a community such as EAACI, operating within a specific domain, it was preferred to simplify the process. It must be noted however that there is the notion of Task

\footnotetext{
${ }^{5}$ http://www.eaaci.net
}

Forces, which can be considered to implicitly contain the concept of topic. Task Forces are groups of individual users and are meant to accommodate special interest groups within EAACI.

The problem formulation phase however corresponded directly to the EAACI guideline decision making process, as it involves formulating a specific issue for which a guideline needs to be authored. Adopting our terminology to IBIS, we chose to refer to problems as Issues in this context. Issues are formulated and shared within a Task Force by the Task Force moderators (using a simple form web interface for this purpose) and remain open for input for a predefined period. This open for input period is broken down to a debating period (idea management phase) and a voting period (voting phase).

The idea management phase was also a natural match to the EAACI guideline decision making process, as for each formulated issue members may either create their own positions (limited in number in order to prevent abuse), or participate by engaging in conversation with other members, commenting and stating opinions on the advantages and disadvantages of existing positions. In order to take advantage of increasing user familiarity with SM, we chose to utilize a set of well-known SM features for collecting feedback, aligned with the IBIS philosophy: simple, yet capable of capturing rich dialogue semantics and structure. Users are able to give feedback in one of the following ways:

1. Add note. Notes are meant to provide additional information in the context of what is being discussed, and can be attached to positions, other notes, as well as arguments for/against.

2. Add argument for. Arguments for are meant to provide additional support for something that has been said in the discussion, and can be attached to positions, notes, as well as other arguments for/against.

3. Add argument against. Arguments against are meant to provide justification for refuting something that has been said in the discussion, and can be attached to positions, notes, as well as other arguments for/against.

4. Rate. Ratings are meant to enable users to take a stance for or against something that has been said in the discussion, and can be applied to notes and arguments for/against.

5. Vote. Votes are meant to provide evaluation of candidate positions using a range voting system ${ }^{6}$.

The voting phase has been shifted in the EAACI guideline decision making process as follows: while an issue remains open for debate, task force members may create positions, add notes and arguments for/agains and rate - all part of the idea management phase. Voting on positions takes place after the debating period ends, during the voting period. During the voting phase users can vote on as many positions as they like, giving each a score in a predefined range (0-10). Users can only vote once for each position, altough they are free to change their vote as many times as they wish for the duration of the voting period.

\footnotetext{
${ }^{6}$ http://www.rangevoting.org
} 
My Consensus

My Consensus | Classification of pediatric rhinitis | $\triangle$ oкıнaoтıкó Topic

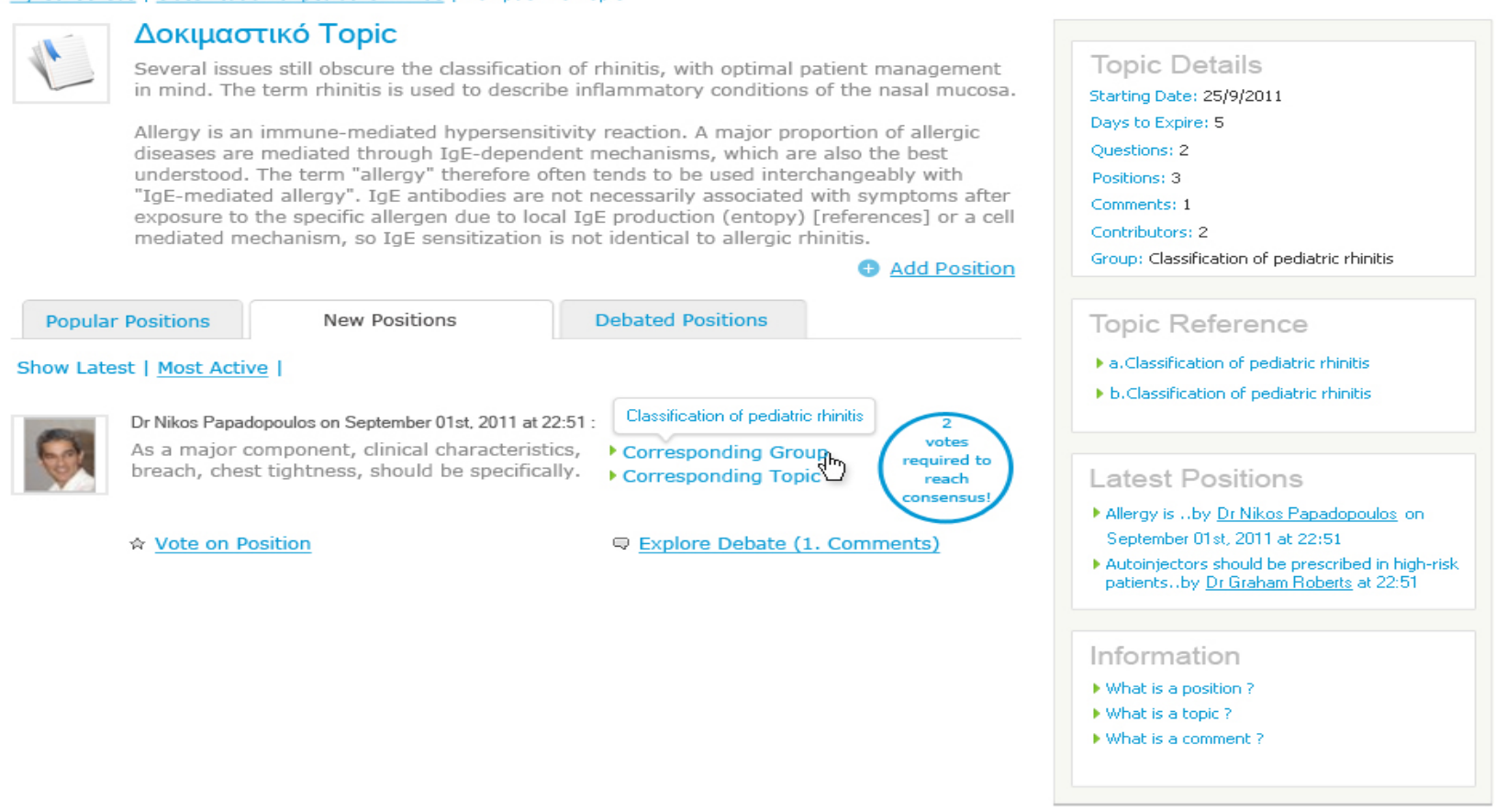

Figure 3: Issue view

This shifting also reflects the fact that the proposal writing process is currently out of scope of the EAACI guideline decision making process, so the voting takes place after the last phase where user input is available. The proposal composition phase will be introduced in the process and the platform as well, after a startup period during which platform users will become accustomed to the basic decision making operation. The goal is to semi-automatically compose proposals based on the highest-rated positions and then validate them via a brief feedback and voting phase as well.

The resulting process is depicted in Fig. 2. In Fig. 3 we show a screenshot from the platform, in which an Issue view during the debating period is displayed. We can see the description of the Issue, as well as additional information, Issue references, the Issue activity stream, the positions expressed for it as well as the argumentation graph for one of these positions.

In order to model our domain appropriately before embarking on platform design and development, the theoretical background of Section 2 was considered and a conceptual alignement was performed, resulting in the definition of the domain and its main concepts. These were captured by means of a domain ontology (see Section 4), which was used as the focal point of an ontological model driven architecture. The platform was developed using standard web application architecture and tools, so there is a data storage layer for which a relational database is used, an object-relational mapping layer that abstracts the storage layer, an application logic layer that exposes Service APIs and a GUI layer that uses these APIs to build the front-end of the platform.

Additionally however, a mapping of the data storage layer to the domain ontology definition was performed, so that deliberation data can be exposed in a semantically well-defined format, as per the Linked Data principles. The mapping was performed using the D2R tool, providing an interoperability layer that enables semantic interoperability with other applications in the domain: deliberation-related data can be exchanged directly via a SPARQL endpoint.

Furthermore, the conceptual model was used as the basis for the design and implementation of a model that estimates argument strength and agreement level based on user feedback: the consensus rate model. As the crystallization of the conceptual model was the starting point for our design, this enabled us to work in parallel on the platform implementation and the the consensus rate model design, integrating it in the platform as the final step of its development. The consensus rate model has been implemented as a distinct module in the platform application depicted in Fig. 4, exposed via the Service Layer. 


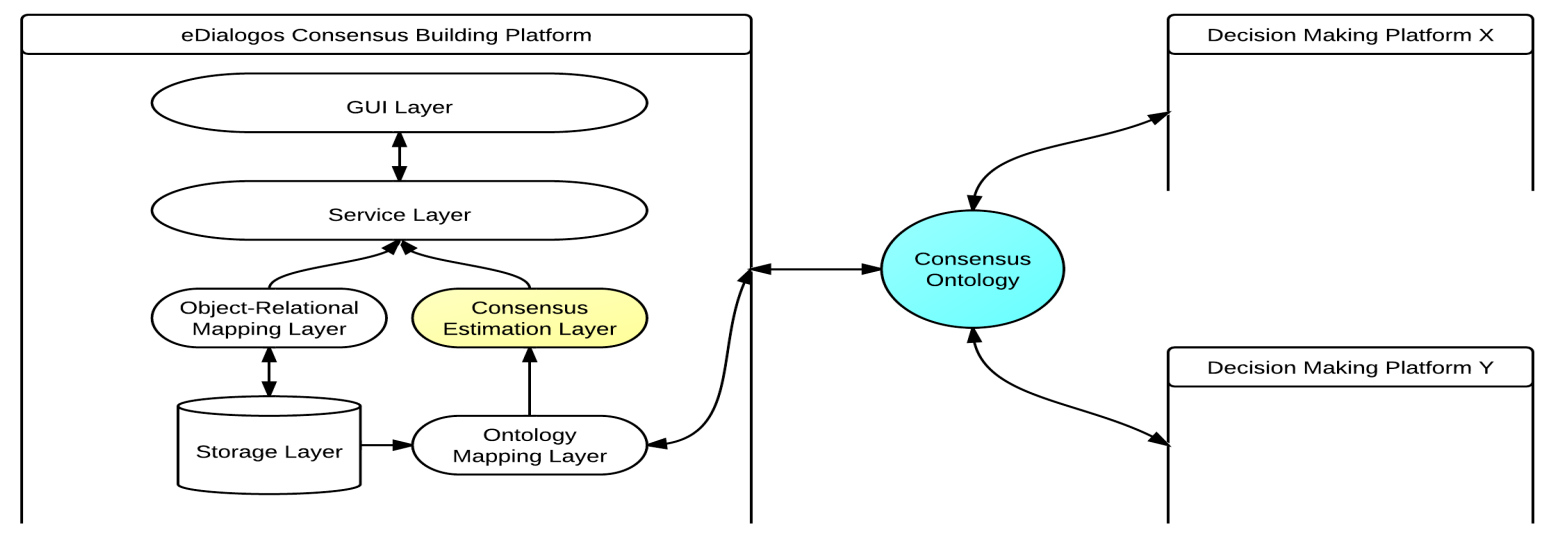

Figure 4: Consensus platform architecture

\section{SEMANTIC WEB TECHNOLOGY TO FA- CILITATE COLLABORATIVE DECISION MAKING: THE EDIALOGOS CONSEN- SUS ONTOLOGY}

Following the definition of the platform architecture, one of the requirements for the deployment of the system at EAACI was to ensure interoperability on data level with other deliberation models (see Section 2), including deliberations as modelled in the previous version of the eDialogos platform. In addition, the data had to be easily publishable and portable to allow tool-independent analysis. Due to the intensive use of Web technologies in the implementation, we have chosen to design the entire data back-end following Semantic Web data modelling principals.

To the best of our knowledge, currently available formalizations of deliberation models do not provide a single ontology that would cover all the concepts and mechanics that were required for the Consensus platform. Therefore, we have created an ontology that integrates contemporary achievements in the domains of Problem Modelling, Idea Management and Collective Decision Making. In the following we elaborate on the manner in which the corresponding ontologies (see Table 1) were integrated. More specifically, we describe the new concepts that the Consensus ontology introduces in order to facilitate the interoperability between the domains of Idea Marketplaces, Idea Management Systems and Issue-Based Information Systems.

To establish the core of the Consensus platform and facilitate the aforementioned integration between different mod- els, the eDialogos Consensus ontology defines three main classes: Argument Map, Issue and Position. Additionally to represent discussions over ideas we introduced: Note and Argument concepts. Each of those classes inherit from a number of classes of external ontologies that focus on modelling respective concepts (see Fig. 5):

- consensus:Issue is primarily based on ProblemChallenge concept that allows definition of various problem types and statuses. In addition since we recognized IdeaContests as a similar concept on the Idea Management side, inheritance from both allows smooth reuse of the Gi2MO ontology properties to integrate both models. Issue also inherits from the IBIS ontology Question concept, as it is the starting point for the decision making process.

- consensus:Position is based on the Idea concept from the Gi2MO ontology. Positions also extend ibis:Idea, as they correspond directly to this concept. Having a concept that inherits from both aforementioned ones ensures a consistent modeling that maintains links with both approaches, while clearly separating from the somewhat unintuitive modeling of the IBIS ontology that bases its whole concept hierarchy on the Idea concept. The new class enables categorization using SKOS controlled vocabularies, as well as free-text annoatation using tags, expressed via the SCOT ontology. It also allows direct relationships with ArgumentMaps that do not have their counterpart in Gi2MO ontology.

- consensus:ArgumentMap expresses the concept of

Table 1: Consensus ontology imports

\begin{tabular}{|l||l|}
\hline Ontology & Description of concepts modelled \\
\hline Dcterms & Generic properties for many assets, e.g. 'title', 'description' etc. \\
\hline Foaf & Relation between User Account in the deliberation platform and personal data \\
\hline Scot & Tags and tagging activities \\
\hline Problem Challenge Ontology & Problem concept, its changes over time and challenge marketplaces model \\
\hline IBIS Ontology & Basic deliberation cycle model, relationships between ideas and associated discussions \\
\hline eDeliberation Ontology & eDeliberation concept \\
\hline Gi2MO Ontology & Idea, Idea Contest, concepts related to expert and collaborative review process \\
\hline
\end{tabular}




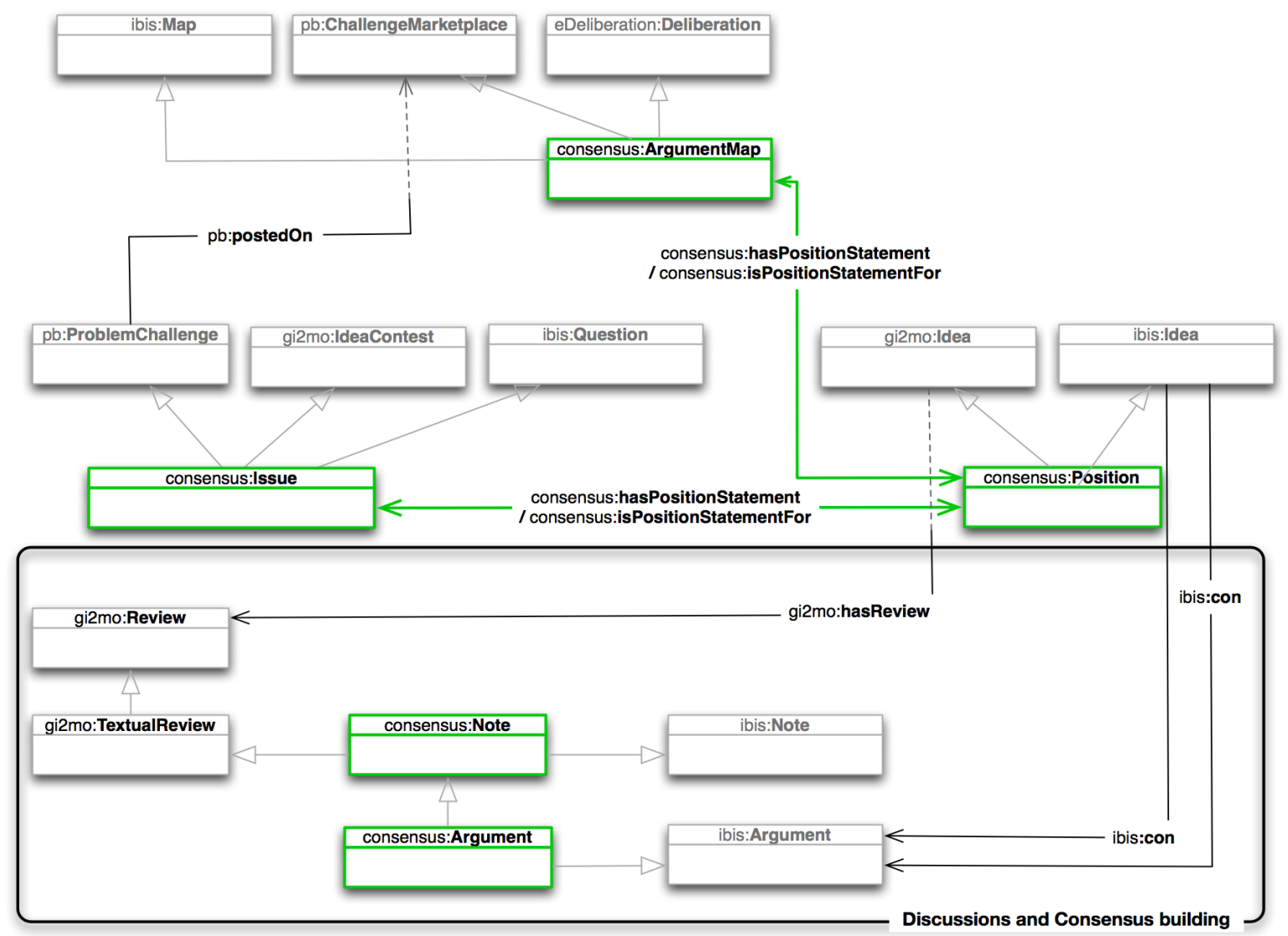

Figure 5: Integration of various ontologies for the eDialogos Consensus model

a common space for sharing all content generated as a by-product of a deliberation process, as described in the eDialogos deliberation ontology [4]. This concept also corresponds directly to the Map concept of the IBIS ontology, shared by all argument mapping tools. In addition, to link it with the problem concept in Idea Marketplaces we model the meaning of Argument maps as an extension of ChallengeMarketplaces from the ProblemChallenge ontology

- consensus:Note and consensus:Argument - both of those concepts relate to the deliberation model presented by IBIS. Notes are the base concept for Arguments, since they both have content and are attached to Positions and Notes and Arguments (using the ibis:refers to and ibis:pro/ibis:con properties for Notes and Arguments respectively). In addition, to connect those concepts to the Idea Life Cycle of Idea Management Systems (and the gi2mo:Idea class) we interpret this part of the IBIS workflow as the Idea Selection stage of the Idea Life Cycle [28] which is represented in Gi2MO ontology as Idea Review.

On top of the above relations in the eDialogos Consensus model we extensively reuse elements of the Gi2MO ontology to represent: gi2mo:Users as creators of any of above concepts, gi2mo:UserGroups to represent Task Forces deliberating around selected topics aggregated with ArgumentMaps.
The categorization and organization of data is modelled using the skos:Concept and scot:Tag formalizations for controlled vocabularies and free-text annotation respectively.

The ontological infrastructure of the eDialogos Consensus platform served a number of purposes. In addition to driving the Model-Driven Architecture for the platform and serving as the interoperability layer with other solutions in the domain, in parallel it facilitated the development of the argumentation and feedback based consensus rate estimation model outlined in the following section.

\section{ARGUMENTATION GRAPHS AND USER FEEDBACK TO ESTIMATE AGREEMENT: THE CONSENSUS RATE MODEL}

One of the design goals for the Consensus platform was pragmatic, functional support for argumentation and debate, in a way that is easy to use for the average web user, and yet can provide tangible benefits in the decision making process. Following the conceptual design of the domain, we incorporated support for argument graphs (via the consensus:Argument concept and the ibis:for and ibis:con properties) that can be attached to Positions as well as other Arguments. We also support the incorporation of user feedback on these Arguments in the form of thumbs up/thumbs down instant reactions. The crystallization of the domain as an ontological model was the basis that enabled us to work 
in parallel on application and model development.

Our goal was not to develop a model for the detection of exceptional content/events, but rather to combine argument graph structure and user feedback to calculate a consensus rate that expresses the agreement level for topics under debate. This is orthogonal to the exception detection logic of [17], which in our case is partially supported by the activity stream updates generated for each user. The model was developed through a process that combined intuition from our domain experience and experience documented in related work. Arguments are viewed as nodes in a graph formed by their relation to each other, and for each node in this graph a consensus rate expressing public degree of agreement is calculated, based on node feedback (for leaf nodes) as well as the propagated consensus rate of the nodes connected to it in a recursive fashion (for parent nodes). In the following we outline the consensus rate model through a series of examples.

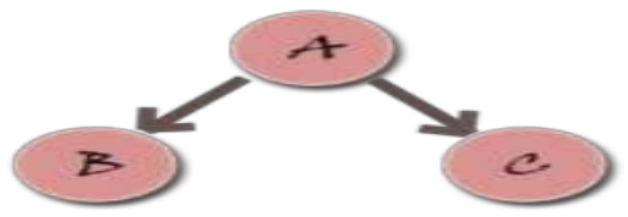

Figure 6: Baseline consensus rate calculation

We start by defining the consensus rate for leaf nodes. In Fig. 6, we have a parent node $\mathrm{A}$ with two leaf nodes, B and C. Given that node C (similar for node B) is an argument for or against node $\mathrm{A}$, users are able to agree or disagree with the argument by rating it with thumbs up or thumbs down respectively. We hereby describe a recursive model over the height of the argumentation tree. Initially, we compute absolute agreement of an argument in the leaves level which will be the base case of the recursion. That is, given a parent node $\mathrm{A}$ that is a thesis (argument support or attack) and two children arguments B, C (again, either in support or attack) we first calculate the agreement level for each node. This calculation is based on the thumbs up and down votes for each node and is expressed as follows, as the rate of absolute agreement:

$$
r_{a b s}(m)=\frac{\sum_{v \in \operatorname{votes}(m)} v}{\sum_{v \in \operatorname{votes}(m)} 1}
$$

The rate of absolute agreement of a node is the ratio of the summary of agreement, to the number of votes. This ranges from -1 to +1 , where values greater than 0 indicate acceptance of the argument while values less than 0 indicate dissaproval. For example, if 23 people disagree and 5 agree with some argument, we get an absolute agreement of $\frac{-18}{28}=-.0 .64$. This score is characterized as absolute due to the local nature of the denominator. This is more clearly explained in Fig. 6, where we assume that nodes B $\& \mathrm{C}$ are populated with thumbs up and down votes. Given that one person agrees and two disagree with $\mathrm{B}$, we get a rate of -0.33 . On the other hand, if 300 people agree and 400 people disagree with $\mathrm{C}$, we get a rate of -0.14 , which is significantly lesser. Intuitively, we would expect to have a greater rate for $\mathrm{C}$, as in this case we have more user input and therefore the public opinion extracted by this is more reliable. Therefore, this a rather misleading result, in the sense that it does not take into account contribution to the comment voting. This missing factor is defined as a second metric, the Relative Rate of Agreement:

$$
r_{r e l}(m)=r_{a b s(m)} * \frac{\sum_{v \in \text { votes }(m)} 1}{\sum_{k \in \text { nodes }(\text { Issue })} \sum_{v \in \text { votes }(k)} 1}
$$

Relative Rate of Agreement for some node $m$ is defined to be the rate of absolute agreement for $m$, multiplied by the participation in that node. Participation normalizes over all node descendants of an Issue (all the arguments in this Issue) and thus provides a more relative perspective. Applying in the previous example where B had 3 votes and B had 700 , the relative agreement is computed as:

$r_{\text {relative }}(B)=\frac{-1}{3} \frac{3}{703}=-0.0014$, and

$r_{\text {relative }}(C)=\frac{-100}{700} \frac{700}{703}=-0.14$

That is, the contribution factor degrades the rate of node $\mathrm{B}$ while leaving almost intact the rate of node $\mathrm{C}$. The relative rate of an argument measures how much do people agree with it (thumbs up and down), out of all the people who could have agreed and it ranges from -1 to 1 , the same range as the absolute rate. Such a measure is important in order to promote arguments that generate lots of feedback. This score may be used recursively upwards the tree, to measure the rate of parent arguments. In the following we define the recursive rate of a node $m$, based on the hypothesis that all of node children are already recursively rated:

$r_{r e c}(m)=a_{1} r_{r e l}(m)+a_{2} \frac{\sum_{c \in \text { children }(m)} r_{r e c}(c)\left(r_{r e c}(c)-e\right)^{2}}{b+\sum_{c \in \text { children }(m)} 1}$

Variables $a_{1}$ and $a_{2}$ are weights defined manually. The sign of both weights reflects the type of the argument. That is, $a_{1}, a_{2}>0$ if argument is for and $a_{1}, a_{2}<0$ else. Variable $a_{1}$ acts as a weight of the relative agreement in the node defined in (2) while variable $a_{2}$, weights the rate coming from the descendant subtrees.

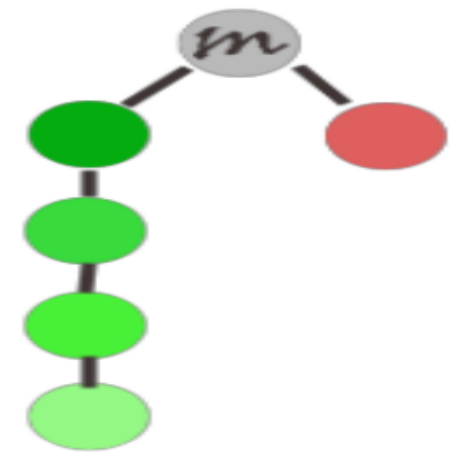

Figure 7: Degradation of argument strenght for deeply rooted arguments by colluding users

The rate from the descendants is defined to be the sum of children rates, scaled by how much each rate deviates from the mean $\frac{\sum_{c \in \text { children }(m)} r_{r e c}(c)}{\sum_{c h i l d r e n}(m)}$. In our case, this scaling factor awards scores which deviate more than normal as these 
should be relatively important. The outcome is normalized with the number of children and smoothened by a variable $b$. This variable only affects the case of deeply rooted arguments with single parent nodes (see Fig. 7). If this is zero, the root of a chain sub-tree created by single parent arguments (left child of node $\mathrm{m}$ ) receives a high value on the second factor of the right part of the equation. This means that, deeply rooted and highly evaluated arguments are important. Else, if this is non zero, this factor degrades to its way up. This is useful to avoid the effect of colluding users. Such a user could create o chain of mutually reinforcing arguments for, in order to promote a root node. This is shown in Fig. 7, in which the shade of green for each node in the chain represents the recursive rate given to the node according to the convention that a darker shade of green stands for a higher rate. Since a chain like this one would not receive a high relative rate (assuming that such a scheme would not be supported by a substantial percentage of users), the last node is colored in light green. However, while going up, the smoothing parameter acts as a control in order to penalize the chain argumentation.

\section{CONCLUSIONS AND OUTLOOK}

We have presented the eDialogos Consensus process and platform, as derived from our own field experience and previous work in the domain of deliberation and collective decision making as well as other existing approaches. Our goal was to develop a process and platform that is easy to use and pragmatic, while also incorporating features that try to address gaps in the applicability of existing approaches. At the same time we wish to adopt these features of existing approaches that have shown potential and maintain compatibility with them, offering an aproach that can also act as a bridge for existing ones. We have tried to achieve these design goals via a modular design and user-friendly interface, adoption of a common methodology and its concepts, and our two contributions to the field:

- The development of the infrastructure for a conceptual and technical bridge among various decision making systems, by means of the eDialogos Consensus ontology.

- The development of a metric that combines argumentation structure with user feedback to provide an indication on the level of agreement and the strength of arguments on topics under debate, by means of the eDialogos Consensus rate.

The result has been implemented and is about to be deployed in order to constitute the decision making and deliberation backbone for the EAACI medical association. An initial beta testing phase has been rolled out in mid-February of 2012 and is available at http://consensus.imc.com.gr. After the beta testing phase has been completed, we will proceed to the full deployment of the platform in its own space and roll out an outreach campaign for EAACI members. Via this process, we will gain additional experience and feedback that will allow ut to develop the process and the platform further in the future. Some of the items we have already identified as future work are listed in the following paragraphs.

Argument map visual representation. One part of the typical IBIS process we have not yet incorporated is the visual representation of argument maps. The rationale for this decision was mainly from a usablity point of view: we wanted to ensure a progressive transition for EAACI users from the current decision making process to an IBIS-based one. Since most users are already familiar with the kind of Web2.0 features we have incorporated in our implementation, we believe the entry barrier will be minimal. After a initial familiarization period, we will introduce a 2-way interpretation process through which dialogue and argumentation graphs developed via the debating process will be visualized in a way similar to the typical visulization paradigms incorporated in other IBIS-based systems. In our case however we will also introduce the additional insight of user feedback and consensus rate in the visulization. Since the starting point for this is also the conceptual model of the Consensus ontology, we have already produced the specifications for this 2-way visualization module and are moving forward with the implementation.

Development and integration of a strategic user participation and engagement game. We believe that encouraging and rewarding constructive participation is of the utmost important for the operation of any community, as it promotes the sense of belonging and equality and helps the common setting of ground rules via social mobility. In this respect, we deem the principles of democratic meeting techniques and normative design (as applied e.g. in [15]) as the guidelines for the development and application of such a game model approach. Again, for the case of EAACI we intend to introduce such a strategic game that rewards user actions according to a participation model, after consulting with EAACI in order to determine the kind of evaluation and reward that is desirable from an organizational point of view. From a conceptual and technical point of view, we deem this process to be well-grounded.

Proposal drafting. The Open Innovation deliberation process includes support for a proposal drafting, which we consider an important part of the process as it is meant to consolidate input received during the initial phases of the process in order to compose a text that captures the collective consensus on the subject under debate. In order for this phase to be introduced into the platform, issues of organizational and cultural as well as of technical nature have to be dealt with. As is usually the case, perhaps the greatest challenge is on the organizational and cultural side, as the idea of direct involvement in the composition of proposals has to grow within organizations. To this end, the EAACI use case is a proof of concept to investigate whether the introduction of a non-mediated involvement in the decision making process, properly supported methodologically and technically, can lead to the development of a participatory culture. On the technical side, techniques for text summarization and composition combined with metrics to incorporate user feedback can be used to this end.

Scaling. What could perhaps be called the final frontier for collective decision making is scaling up, as this has been one of the main obstacles for applying such techniques in a wide array of contexts, including political decision making. While the use of an ICT backbone can ensure the technical capability to accommodate the load this entails, the infrastructure alone does not suffice to make this a reality without an appropriate architecture. In this respect, we deem the Outcast voting network architecture (see Fig. 8) an important step to that direction and are looking into ways of 
integrating with it, as the two approaches are orthogonal. Outcast defines an architecture for a voting infrastructure with the aim of scaling to communities that are large in number and self-organised in nature. Outcast also supports the notion of vote delegation and is a sophisticated architecture for the provision of an integrated environment for Adhocracy [20]. However at this stage the architecture focuses on the voting part, and even though there is an outline for the integration of drafting and discussion media, this is left unspecified. We believe therefore that this work complements the architecture in a very suitable way, not only by providing instances of such a drafting and discussion medium, but also by semantically specifying the integration points and process.

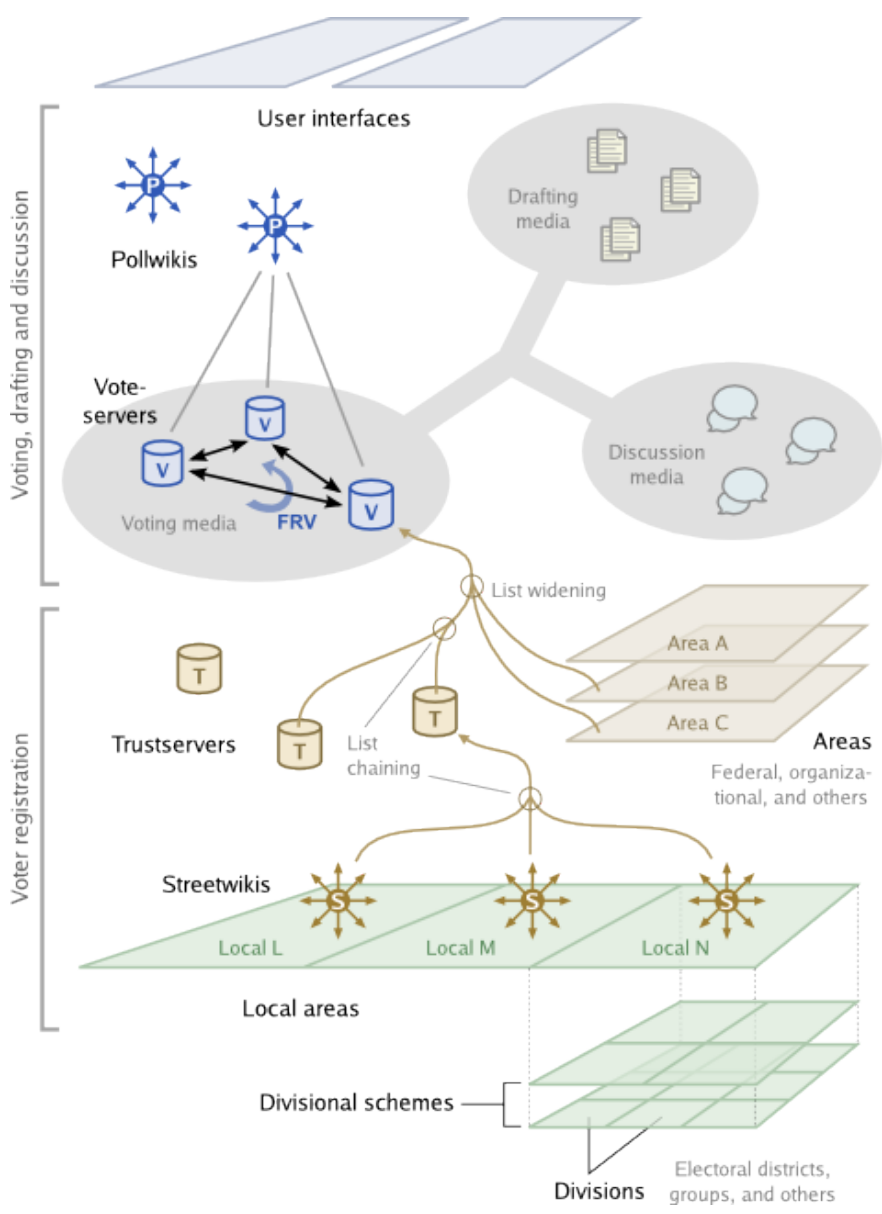

Figure 8: Outcast voting network

Argumentation grounding. Although we have chosen not to pursue an approach geared towards formal argumentation, as this would discourage widespread use and adoption of our process and platform at this stage, we do not underestimate its potential. One of our goals therefore is to work on the integration of formal argumentation in the proposed framework, enabling the expression of abstract argumentation by users who are willing and capable to use this formalism.

Availability. Last but not least, in order for the eDialogos Consensus platform to achieve maximum effect and be used where it is really needed, we are planning to make the core of the platform available under a dual licensing mode that will ensure free use for non-for-profit causes and organisations. The eDialogos Consensus ontology is already available at http://www.imc.com.gr/ontologies/eDialogos/consensus under a Creative Commons License.

We see this as an ongoing multidimensional process, one that combines literature and scientific work, technological progress and social reflection in order to provide an evolving aproach to wide-scale collaborative decision-making. We hope that the approach will be succesful and useful for future work of our own as well as others in the field.

\section{ACKNOWLEDGMENTS}

We would like to thank Dr. Nikos Papadopoulos from EAACI for supporting this work and providing valuable insight in the inner working and structure of EAACI and its decision making process. We also would like to thank the eDialogos Consensus platform development team, namely Manolis Georgopoulos, Thodoris Germanis, Maria Melabioti and Aristotelis Zosakis that made the implementation of the platform possible, as well as Marc-Antoine Parent for the discussion and insights on the Open Innovation deliberation process.

\section{REFERENCES}

[1] CROSSROAD Project. Deliverable 2.1: State of the Art Analysis. http://crossroad.epu.ntua.gr/ files/2010/04/CROSSROAD-D1. 2-State-of-the-Art-Analysis-v1.00.pdf, 2011. [Online; accessed 17-2-2011].

[2] Outcast Voting Network. http: //zelea.com/project/outcast/__overview.xht, 2011. [Online; accessed 17-2-2011].

[3] G. Anadiotis, P. Alexopoulos, K. Kafentzis, E. Konstantinou, M. Stankovic, and M. Waeckerle. Semantics-powered Virtual Communities and Open Innovation for a Structured Deliberation Process. In Workshop on Semantics for Governance and Policy Modelling, ESWC, 2011.

[4] G. Anadiotis, P. Alexopoulos, K. Mpaslis, A. Zosakis, K. Kafentzis, and K. Kotis. Facilitating Dialogue Using Semantic Web Technology for eParticipation. In Extended Semantic Web Conference (ESWC), 2010.

[5] D. Ayers. IBIS Vocabulary. http://hyperdata.org/xmlns/ibis/, 2006. [Online; accessed 17-2-2011].

[6] V. Belak and V. Svatek. Supporting Self-Organization in Politics by the Semantic Web Technologies. In 2nd International Conference on eParticipation (ePart), 2010.

[7] N. Benn and A. Macintosh. Using PolicyCommons to support the policy-consultation process: investigating a new workflow and policy-deliberation data model. In Modelling Policy-Making (MPM), 2011.

[8] R. Boero, E. Ferro, M. Osella, Y. Charalabidis, and E. Loukis. Policy Intelligence in the Era of Social Computing: Towards a Cross-Policy Decision Support System. In Workshop on Semantics for Governance and Policy Modelling, ESWC, 2011.

[9] H. Chesbrough. The open innovation paradigm. Open innovation: The new imperative for creating and 
profiting from technology.

[10] P. Ciccarese, E. Wu, J. Kinoshita, G. Wong, M. Ocana, A. Ruttenberg, and T. Clark. The SWAN Biomedical Discourse Ontology. Journal of Biomedical Informatics, 41(5):739-751, Oct 2008.

[11] J. Conklin. Dialogue Mapping: Building Shared Understanding of Wicked Problems. John Wiley and Sons, Ltd., 2005.

[12] J. Conklin, A. M. Selvin, S. B. Shum, and M. Sierhuis. Facilitated hypertext for collective sensemaking: 15 years on from gIBIS. In HYPERTEXT 2001, Proceedings of the 12th ACM Conference on Hypertext and Hypermedia, 2001.

[13] A. de Moor, J. Park, and M. Croitoru. Argumentation Map Generation with Conceptual Graphs: the Case for ESSENCE. In 4th ICCS Conceptual Structures Tool Interoperability Workshop (CS-TIW), 2009.

[14] T. F. Gordon. The Policy Modeling Tool of the IMPACT Argumentation Toolbox. In Modelling Policy-Making (MPM), 2011.

[15] K. Hansson, P. Karlstrom, A. Larsson, and H. Verhagen. Actory: A tool for visualizing reputation as a means to formalize informal social behavior. In 2nd International Conference on Reputation: Society, Economy, Trust (ICORE, 2011.

[16] N. Karacapilidis, M. Tzagarakis, N. Karousos,

G. Gkotsis, V. Kallistros, S. Christodoulou, and C. Mettouris. Tackling cognitively-complex collaboration with CoPe_it! International Journal of Web-Based Learning and Teaching Technologies, 4:22-38, 2009.

[17] M. Klein. Using Metrics to Enable Large-Scale Deliberation. In Collective Intelligence In Organizations: A Workshop of the ACM Group 2010 Conference, 2010.

[18] J. Leite and J. Martins. Social abstract argumentation. In 22nd International Joint Conference on Artificial Intelligence (IJCAI), 2011.

[19] P.-A. Matt and F. Toni. A game-theoretic measure of argument strength for abstract argumentation. In Proceedings of the 11th European conference on Logics in Artificial Intelligence, 2008.

[20] H. Mintzberg and A. McHugh. Strategy Formation in an Adhocracy. Administrative Science Quarterly, 30(2):160-197, 1985.

[21] E. Panopoulou, E. Tambouris, and K. Tarabanis. eParticipation initiatives in Europe: learning from practitioners. In 2nd International Conference on eParticipation (ePart), 2010.

[22] J. Park. IBIS Common Format. http://projects.kmi.open.ac.uk/hyperdiscourse/ docs/IBIS-0.1.pdf, 2009. [Online; accessed $17-2-2011]$.

[23] I. Rahwan, F. Zablith, and C. Reed. Laying the foundations for a World Wide Argument Web. Artificial Intelligence, 171:897-921, July 2007.

[24] M. A. Rodriguez, D. J. Steinbock, J. H. Watkins, C. Gershenson, J. Bollen, V. Grey, and B. deGraf. Smartocracy: Social networks for collective decision making. In 40th Hawaii International International Conference on Systems Science (HICCS), 2007.
[25] O. Scheuer, F. Loll, N. Pinkwart, and B. M. McLaren Computer-supported argumentation: A review of the state of the art. I. J. Computer-Supported Collaborative Learning, 5(1):43-102, 2010.

[26] S. B. Shum. Cohere: Towards Web2.0 Argumentation. In 2nd International Conference on Computational Models of Argument (COMMA), 2008.

[27] R. Siebes. Parademo: e-Democracy Based on a Delegated Expert Selection Process in a Small-World Network. In e-Democracy'09, 2009.

[28] A. Westerski, C. A. Iglesias, and T. Nagle. The road from community ideas to organisational innovation: A life cycle survey of idea management systems. $A$ Special Issue of the Journal Web-Based Communities. Community-based Innovation: Designing Shared Spaces for Collaborative Creativity., 2011.

[29] A. Wyner, K. Atkinson, and T. Bench-Capon. Semantic Models and Ontologies in Modeliling Policy-making - A Position Paper. In Modelling Policy-Making (MPM), 2011. 